\title{
A Quantitative Evaluation of Haptic Data Prediction Techniques over Best-Effort Network
}

\author{
Tiam Hee Tee*, Kian Meng Yap*, Alan Marshall**, Kok Seng Eu* \\ *Department of Computer Science and Networked Systems, Sunway University, Selangor, Malaysia \\ **School of Electrical Engineering, Electronics and Computer Science, University of Liverpool, Liverpool, United Kingdom \\ 12056644@imail.sunway.edu.my,kmyap@sunway.edu.my, alan.marshall@liverpool.ac.uk, 12058999@imail.sunway.edu.my
}

\begin{abstract}
Exchanging haptic information over best-effort networks such as the Internet presents challenges due to the extremely high sensitivity to network impairments, especially the simultaneous occurrence of time-varying network latency and packet loss. Subsequently, the haptic interaction experience is deteriorated along with a reduction in the fidelity. In this paper we present a new approach to mitigate the effects of network impairments, termed Trust Strategy Prediction. As well as evaluation of TSP in quantitative model is presented in terms of accuracy and smoothness of haptic updates and compared with two well-known techniques used in haptic data prediction: Dead Reckoning and velocity/yank Estimation with filtering mechanism.
\end{abstract}

Keywords - Haptics, tele-operation, haptic predictive control, communication network, trust strategy prediction.

\section{INTRODUCTION}

Performing haptic interactive application over the Internet enables users to exchange haptic data through the Internet service providers. Haptics require laws or policies to specify the action and reaction between force (i.e. force with Cartesian vector) and motion (i.e. kinematics with Jacobian transpose) [1]. Therefore, haptic data involves positions, angles, velocities, forces and torques, which demands stringent requirements in terms of network time latency and packet exchange rate [2]. Such real-time, extremely high transmission rate and loss-sensitive nature of haptic interactive applications poses a serious challenge whenever haptic data is transmitted over the best effort network (i.e. the Internet), which is a nonadministrative communication channel and varies over time according to the network conditions. Conventionally, haptic type of network traffic is not prioritized but shares the same network bandwidth with many other types of network traffic (i.e. text, graphics, audio and video). Unlike the administrative network with quality of service (QoS) which allows configuring the specific type of traffic to have privilege treatment and higher priority of network propagation [3], besteffort network is unavoidable to be influenced by the network impairment. For example, haptic interactions can be simultaneously affected by packet loss and network latency with jitter over the Internet. It will eventually lead to poor experience, instability and low fidelity in haptic interaction.
The solutions to mitigate against latency and packet loss in networked haptic systems have been proposed by using approaches consisting of selection of Internet protocols [4], network with QoS [3], control system techniques [5], buffering control [6] and signal processing algorithms [7]. These approaches mainly aim to minimize the negative experience in haptic interaction due to the network impairments. In addition, these approaches tend to be used together with data prediction techniques in order to further ensure the stability and optimize fidelity. One common approach to deal with the issue of real-time interactions that are affected by network impairments is to compensate using prediction mechanisms [8]. In haptic operation, the aid of prediction algorithm attempts to forecast the haptic data based on historical data and then apply the predicted data before the actual data is being received. On the other hand, the evaluations of haptic experiences with proposed mechanisms or frameworks generally involve subjective assessments using Mean Opinion Scores (MOS), task completion time or a haptic perceptibility rating. As such, the human-perception qualitative analyses can indicate which mechanisms or frameworks are working well as compared to others. However, it does not clearly, or quantitatively, show how well the mechanisms or frameworks are performing.

In this paper, an evaluation using a quantitative analysis model in terms of accuracy and smoothness of haptic updates is presented for TSP and it is compared with two common prediction techniques applied in haptic data processing: Dead Reckoning (DR), and velocity/yank Estimation with filtering mechanism $(\mathrm{E}+\mathrm{F})$. The quantity of haptic experience in the aspects of accuracy and smoothness haptic update are evaluated in our quantitative analysis. The accuracy is quantified by the discrepancy value between desired haptic data and predicted haptic data; the smoothness update is quantified by the discrepancy value between the last updated and current update haptic data. This quantitative analysis has presented the human perception with quantified results which illustrate how well the performances of mechanisms or frameworks are, in terms of accuracy and smoothness of haptic update. In addition, the advantage of using quantitative analysis in haptic data prediction is that the maximum 
threshold of network impairments for just-noticeable different (JND) in stability of haptic interaction can be easily identified.

The remainder of this paper is organized as follows: Section II reviews the literature on haptic data processing using prediction approaches and evaluation methods; Section III discusses the TSP framework; Section IV describes the experimental platform and testing scenarios; Section V shows the outcome of the quantitative results and finally the conclusions are presented in Section VI.

\section{Haptic Data Processing Using Predictive ANd EVALUATION APPROACHES}

The key requirement in networked haptic interaction is to achieve realism which has the ability to produce a natural and smooth gesture or body movement, tactile or touch sensory feedback between haptic environments without the restriction of distance. Nevertheless, it recognized that it is difficult to maintain a satisfactory user experience whenever there are network impairments. To mitigate the negative haptic experience caused by network impairments, the current trend of networked haptic research is inclined to incorporate data prediction techniques into the haptic data processing [8].

Many prediction techniques have been applied in haptic interactive applications, especially for those connected through best-effort networks which are simultaneously impaired by network latency and packet loss. Prediction techniques such as deadband [9], linear prediction method [10], DR [11], Smith-prediction [8], Bayesian-prediction[12] and velocity estimation[13][14] have been considered to solve network latency and packet loss. Moreover, to achieve realtime smooth haptic updates, smooth filtering is applied along with the haptic data prediction. Methods such as leastsquares[13], median filtering techniques[14], LyapunovKrasovskii-like function[5] and moving average filter [15] are commonly used to maintain the stability of haptic updates.

In addition, in order to evaluate the performance of proposed mechanisms or frameworks, many haptic researchers tend to make use of subjective assessments to identify whether proposed mechanisms or frameworks are better than others. In [11][13][16][17][18] have carried out users' experience surveys by using MOS and haptic perceptibility rating as the rating of quality of experience (QoE) to subjectively evaluate effect of haptic experience under network impairments. The evaluations on how network impairments affect the haptic experience have shown that impairments lead to unsatisfactory user experience. On the other hand, the evaluation approach used by Osman et al. [22][23] is through assessment of time completion. The faster the completion, the greater is judged the performance of the mechanisms or frameworks.

\section{III.TSP FRAMEWORK}

Due to network impairments, de-synchronization of haptic data between interactive environments has been a major obstacle in achieving realistic of haptic interaction. As a result, operators experience low fidelity in haptic performance, resulting in coarse, irregular movement and abrupt force reflection feedback. To minimize poor user experience due to haptic data de-synchronization, TSP is proposed. TSP is based on historical haptic data and connectivity information such as delay duration and rate of packet loss to predict the haptic data in real-time/current time. It hence contributes to the stability (smoothness update) and realism (accuracy of predicted result) of haptic interactions over best effort networks such as the Internet. The framework proposed in this paper consists of two contributions such as haptic data processing and TSP modelling which will be discussed in section III.A and section III.B respectively.

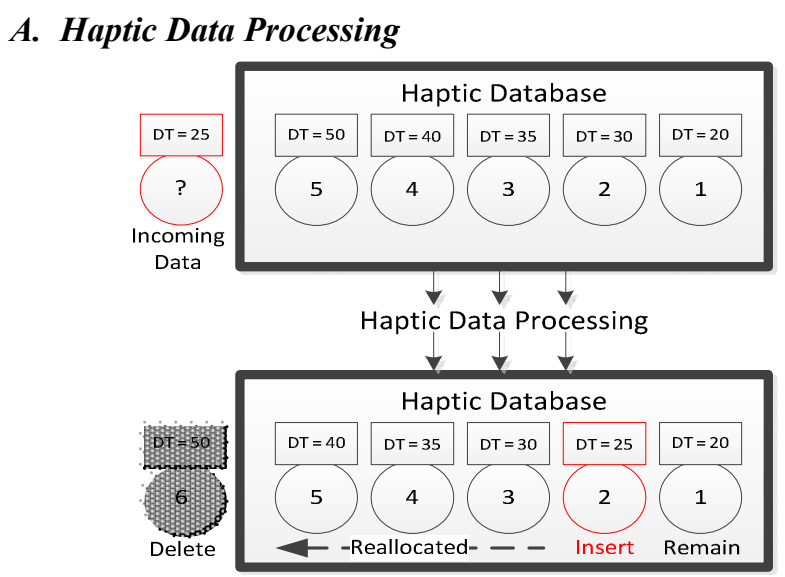

Figure 1. Haptic data processing with relatively recent haptic data

The purpose of this is to receive haptic data from the network and keep the sequence in a timely manner even if the data arrives out-of-sequence. This approach also retains relatively recent haptic data, which is still valid to the prediction, rather than keeping only the very latest data. Upon reception of the data, haptic data processing is performed to ensure the received haptic data is valid and in sequence while maintaining the timeline between haptic environments. In order to maintain timeliness, a timestamp is attached to each haptic packet. At the receiver side, the timestamp can be used to track the current status of network delay $D T$ which is obtained from (1), the difference between the packet received time $R T$ and packet timestamp $S T$.

$$
\mathrm{DT}=\mathrm{RT}(\mathrm{t})-\mathrm{ST}(\mathrm{t})
$$

The verification of valid haptic data is through comparison of the delay between the incoming data and the stored data. Only the five most relatively recent sets of haptic are data retained in the haptic database. For example, Figure 1 presents the scenario when the incoming data has $25 \mathrm{~ms}$ of delay, the haptic data processing first compares its delay with the delay of the fifth haptic data (the oldest data in the haptic database). If the incoming data is more recent than the fifth data, the incoming data is as a valid data and then the fifth data will be deleted otherwise the incoming is discarded. Secondly, the valid incoming data needs to be allocated in the right sequence with a timely concern. In Figure 1, the incoming data is 
allocated between the first and second data because its delay is smaller than the second data and bigger than the first data Then the original second, third and fourth data need to be reallocated with a new sequence of third, fourth and fifth respectively.

With the five relatively new haptic data, TSP then obtains the velocity and yank estimations according to the haptic data by using (2) for position data and (3) for force data.

$$
\begin{aligned}
& \mathrm{V}(\mathrm{t})=\frac{X(t)-X\left(\mathrm{t}_{-1}\right)}{S T(t)-S T\left(\mathrm{t}_{-1}\right)} \\
& \mathrm{Y}(\mathrm{t})=\frac{F(t)-F\left(\mathrm{t}_{-1}\right)}{S T(t)-S T\left(\mathrm{t}_{-1}\right)}
\end{aligned}
$$

where $\mathrm{V}(\mathrm{t})$ and $\mathrm{Y}(\mathrm{t})$ are the velocity (position per unit time) and yank (force per unit time) [21] respectively; $\mathrm{X}(\mathrm{t})$ and $\mathrm{F}(\mathrm{t})$ are the latest position and force value respectively; $\left(\mathrm{t}_{-1}\right)$ which represent as previous notation. It is worth to note that due to the network impairments, the last received data is not necessary to be the most recent data.

\section{B. Trust Strategy Prediction (TSP) Modelling}

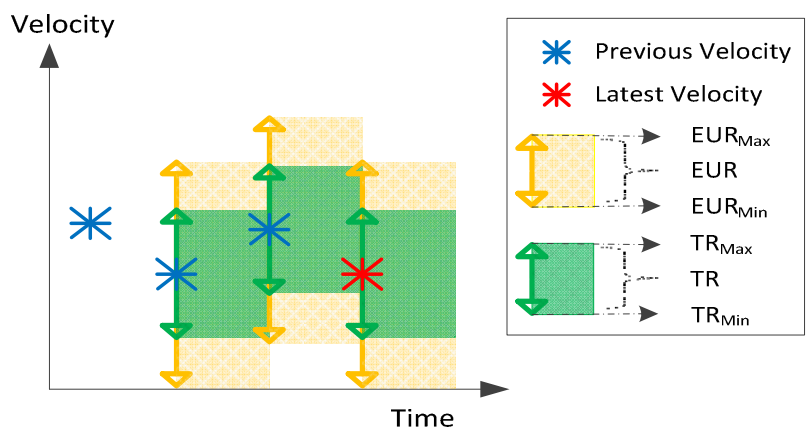

Figure 2. Eligible update range and trusted range obtaining form velocitiy/yank estiamtion.

With the values of velocity and yank estimations, TSP can then start to predict the current status of haptic update. The concept of TSP is based on one of the three behaviours such as untrusted, trusting and trusted behaviour which identified by the latest acquired velocity and yank, is to be applied in the prediction. Each behaviour will have different approach of prediction to estimate the current haptic data. Figure 2 shows that each acquired velocity or yank has its own set of eligible update range $\left(\mathrm{EUR}_{[\mathrm{V}, \mathrm{Y}]}\right)$ and trusted range $\left(\mathrm{TR}_{[\mathrm{V}, \mathrm{Y}]}\right)$ with maximum and minimum values of the ranges that obtain from (4)-(5) and (6)-(7) respectively. With the EUR, the ratios of the ranges are dynamically defined by percentage of the current network delay while $C_{A P}$ in (6) and (7) is the arbitrary prediction constant which has statically set to 0.03 for the best performance in accuracy and smoothness of haptic updates. The value of $\mathrm{C}_{\mathrm{AP}}$ is obtained by several trails and then error tuning under the experimental platform.

After ranges of TR and EUR have been acquired, the process of TSP is then process to provide smooth movement and consistency of haptic update while maintaining the accuracy of haptic data prediction. TSP consists of three prediction behaviours such as untrusted, trusting and trusted behaviour. Each behaviour produces a different predicted result which relies on various network characteristics and historical of haptic data. Every incoming haptic data will have to go through this process and subsequently, the execution of haptic update will be based on the predicted result from a specific behaviour.

$$
\begin{aligned}
& \mathrm{EUR}_{\mathrm{V}}=\left\{\begin{array}{l}
\mathrm{EUR}_{\mathrm{V}_{-} \text {MAX }}=\mathrm{V}\left(\mathrm{t}_{-1}\right)+\left(\mathrm{V}\left(\mathrm{t}_{-1}\right) \times \frac{\mathrm{DT}}{100}\right) \\
\mathrm{EUR}_{\mathrm{V}_{-} \text {MIN }}=\mathrm{V}\left(\mathrm{t}_{-1}\right)-\left(\mathrm{V}\left(\mathrm{t}_{-1}\right) \times \frac{\mathrm{DT}}{100}\right)
\end{array}\right. \\
& \mathrm{EUR}_{\mathrm{Y}}=\left\{\begin{array}{l}
\mathrm{EUR}_{\mathrm{Y} \_\mathrm{MAX}}=\mathrm{Y}\left(\mathrm{t}_{-1}\right)+\left(\mathrm{Y}\left(\mathrm{t}_{-1}\right) \mathrm{X} \frac{\mathrm{DT}}{100}\right) \\
\mathrm{EUR}_{\mathrm{Y}_{-} \mathrm{MIN}}=\mathrm{Y}\left(\mathrm{t}_{-1}\right)-\left(\mathrm{Y}\left(\mathrm{t}_{-1}\right) \mathrm{X} \frac{\mathrm{DT}}{100}\right)
\end{array}\right. \\
& \mathrm{TR}_{\mathrm{V}}=\left\{\begin{array}{l}
\mathrm{TR}_{\mathrm{V}_{-} \mathrm{MAX}}=\mathrm{V}\left(\mathrm{t}_{-1}\right)+\left(\mathrm{V}\left(\mathrm{t}_{-1}\right) \mathrm{XC}_{\mathrm{AP}}\right) \\
\mathrm{TR}_{\mathrm{V}_{-} \mathrm{MIN}}=\mathrm{V}\left(\mathrm{t}_{-1}\right)-\left(\mathrm{V}\left(\mathrm{t}_{-1}\right) \mathrm{X} \mathrm{C}_{\mathrm{AP}}\right)
\end{array}\right. \\
& \mathrm{TR}_{\mathrm{Y}}=\left\{\begin{array}{l}
\left.\mathrm{TR}_{\mathrm{Y}_{-} \mathrm{MAX}}=\mathrm{Y}\left(\mathrm{t}_{-1}\right)+\left(\mathrm{Y}_{\left(\mathrm{t}_{-1}\right)}\right) \mathrm{XC}_{\mathrm{AP}}\right) \\
\left.\mathrm{TR}_{\mathrm{Y}_{-} \mathrm{MIN}}=\mathrm{Y}\left(\mathrm{t}_{-1}\right)-\left(\mathrm{Y}_{-1}\right) \mathrm{t}_{-1} \mathrm{C}_{\mathrm{AP}}\right)
\end{array}\right.
\end{aligned}
$$

\section{1) Untrusted Behaviour}

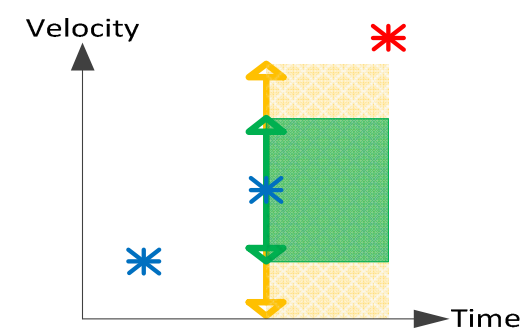

Figure 3. Untrusted behaviour where the new velocity is out of the eligible update range.

The situation of untrusted behaviour is occurred during a large velocity discrepancy between new and previous velocities. This phenomenon could possibly happen due to burst packet loss and a large gap between variable delays and users will immediately feel the abrupt movement and/or force reaction. In addition, it will trigger a large data discrepancy as compared to the source. As Figure 3 shows that the identifier of untrusted behaviour is when the new velocity is out of the range of EUR and TR. Therefore, the new velocity cannot be directly applied into the prediction. The replacement of new velocity $V r$ and new yank $Y r$ are calculated with sample moving average from the three latest velocities as shown in (8) and (9) respectively.

$$
\begin{aligned}
& \mathrm{Vr}=\frac{\mathrm{V}(\mathrm{t})+\mathrm{V}\left(\mathrm{t}_{-1}\right)+\mathrm{V}\left(\mathrm{t}_{-2}\right)}{3} \\
& \mathrm{Yr}=\frac{\mathrm{Y}(\mathrm{t})+\mathrm{Y}\left(\mathrm{t}_{-1}\right)+\mathrm{Y}\left(\mathrm{t}_{-2}\right)}{3}
\end{aligned}
$$




\section{2) Trusting Behaviour}

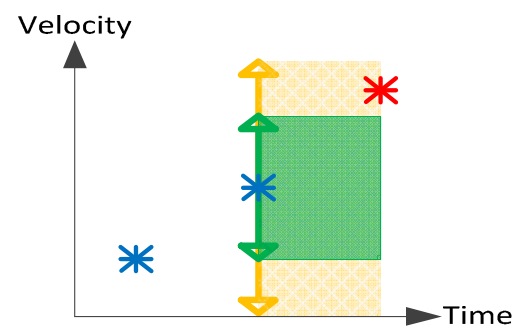

Figure 4. Trusting Behavior where the new velocity is within the eligible update range but out of the trusted range.

The trusting behaviour is used to deal with inconsistencies of smoothness which can be identified when the latest velocity is within the EUR but out of the TR Figure 4 as indicates. With the trusting behaviour, a replacement of the latest velocity is also needed in order to prevent unsmooth update. Unlike the formula used in untrusted behaviour, trusting behaviour applied weighted moving average and the formula is shown in (10) and (11) for position and force data respectively.

$$
\begin{aligned}
& V r=\frac{3 V(t)+2 V\left(t_{-1}\right)+V\left(t_{-2}\right)}{6} \\
& \mathrm{Yr}=\frac{3 \mathrm{Y}(\mathrm{t})+2 \mathrm{Y}\left(\mathrm{t}_{-1}\right)+\mathrm{Y}\left(\mathrm{t}_{-2}\right)}{6}
\end{aligned}
$$

\section{3) Trusted Behaviour}

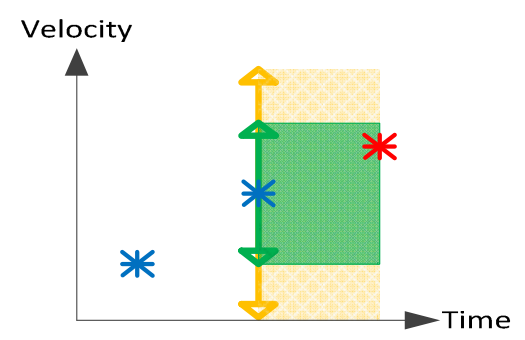

Figure 5. Trusted Behaviour where the new velocity is within the trusted range and it is a valid velocity.

The last behaviour of TSP, trusted behaviour, is a straightforward process to predict the current position and force based on the latest velocity and yank without requirement of $V r$ or $Y r$ when latest velocity value is within the eligible update range and trusted range as shown in Figure 5. After the final velocity is identified and obtained, the predicted results can only be executed by using (12) and (13) for position and force value respectively. $p X(t)$ and $p F(t)$ which are the predicted position and force value in the current time $T$.

$$
\begin{aligned}
& \mathrm{pX}(\mathrm{t})= \\
& \left\{\begin{array}{c}
\mathrm{X}(\mathrm{t})+\mathrm{V}(t)(T-S T(t)), \text { if }(\text { Trusted Bhv. }) \\
\mathrm{X}(\mathrm{t})+\operatorname{Vr}(T-S T(t)), \text { else }
\end{array}\right.
\end{aligned}
$$

$$
\begin{aligned}
& \mathrm{pF}(\mathrm{t})= \\
& \left\{\begin{array}{c}
\mathrm{F}(\mathrm{t})+\mathrm{Y}(t)(T-S T(t)), \text { if (Trusted Bhv. }) \\
\mathrm{F}(\mathrm{t})+\mathrm{Yr}(T-S T(t)), \text { else }
\end{array}\right.
\end{aligned}
$$

\section{IV.EXPERIMENTAL Platform}

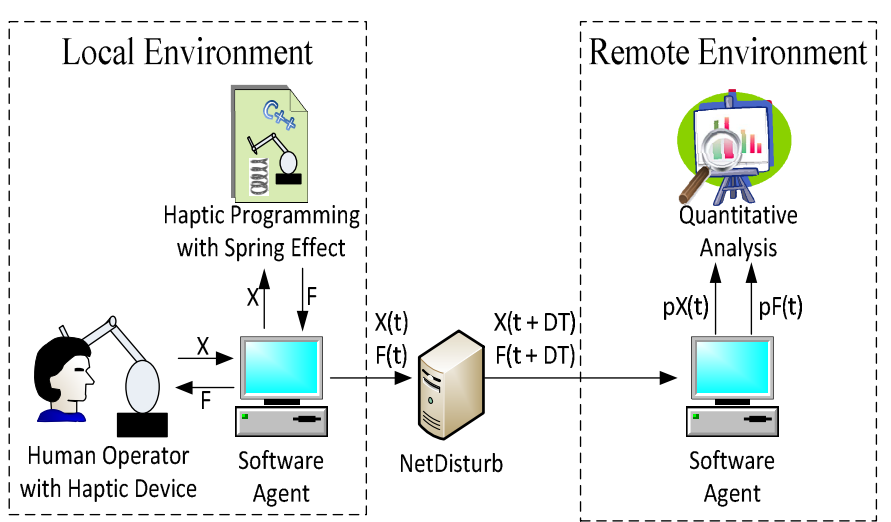

Figure 6. Experimental platform implementation.

The objective of experimental implementation is to study the quantitative analysis with the aspect of accuracy and smoothness in haptic update based on the predicted haptic data which is obtained from the three prediction techniques: DR, $\mathrm{E}+\mathrm{F}$ and the proposed TSP. In terms of accuracy analysis, maximum and average of discrepancy between the haptic data from the transmitter and the predicted haptic data from the three prediction techniques, are compared. This analysis will determine which predictions provide the better accuracy as compared to others. The higher value of discrepancy, the lesser accurate produced by the prediction. As well as smoothness update analysis, maximum and average of haptic update inconsistency which is the absolute difference between the current predicted haptic data and last predicted haptic data, is compared. This is used to quantified and then verify the smoothness of movement. The larger the inconsistency value, the lesser the smoothness of the movement. This will trigger the abrupt vibration feedback if the large gap of inconsistency is updated. In addition, the one advantage of

Figure 6 shows the setup of the experimental platform that used to perform quantitative evaluation. The experimental setup involves two haptic environments: local and remote environment. The local environment consists of a Phantom Omni haptic device [22] as the haptic controller and interconnected to a local software agent which runs on a Core i5 microprocessor with $3.2 \mathrm{GHz}$ clock speed and 16GB DDR3 ram. The remote environment consists of remote software agent running on a Core i3 microprocessor with $3.4 \mathrm{GHz}$ clock speed and 4GB DDR3 ram. Both haptic environments are Internet Protocol network connected via a network emulator called NetDisturb [23] which is used to emulate network impairments onto the traffic flowing. The network emulator runs on a Pentium 4 microprocessor with 2.8GHz clock speed and 512MB SD ram. The software agents 
and network simulator are connected by 1000BASE-T category 5e cables. User Datagram Protocol (UDP) is used as the network transmission protocol for packet exchange, at a rate of 1000 packets/second. During the experiment, a haptic program with spring effect is executed. While the operator is sense the spring effect by using Phantom Omni, the both position and force haptic data are transmitted to the remote environment. At the remote environment, the software agent is then received the incoming haptic data, process the predictions and finally output the predicted result for quantitative analysis.

In this setup, the communication models and their associated parameters have been pre-defined in order to conduct the testing under the Internet-like network impairments of variable delay (including different range of jitter) and packet loss. In order to compare the performance between DR, E+F and proposed TSP, there are 24 sets of testing scenarios (TS) as shown in TABLE 1 to be carried out. TS are based on different packet loss rate settings and various levels of jitter. The percentage of packets lost is configured according to the TS with the burst loss ranging from 1 packet to 20 packets. The NetDisturb applies a continuous uniform distribution [23] to generate the jitter values at random with three defined parameters such as alpha (minimum value of the range), beta (maximum value of the range) and constant delay. Throughout this experiment, the $\mathrm{X}$ axis base joint (known as turret left + ) from the Phantom Omni's, is used as the measurement unit of the predicted response in angular degree $\left({ }^{\circ}\right)$ and force $(\mathrm{N})$.

\section{Performance Analysis and Discussions}

All results are presented from Figure 7 to Figure 10. Two sets of vertical axis from the charts represent maximum value (blue column as primary vertical axis in left) and average (red line as secondary vertical axis in right) values; Horizontal axis represents the three prediction techniques among the 24 sets of TS and the performances of three prediction techniques are presented in every set of TS. Figure 7 and Figure 8 are to show the quantitative analysis in terms to accuracy and smoothness in haptic position update based on the three techniques of prediction.

Figure 7 illustrates the quantitative comparison between the prediction techniques with the aspect of accuracy in position data. The accuracy performance in DR prediction as indicated in Figure 7 is always deviated from the desired position with the highest position discrepancy in both maximum and average quantitative analysis. The accuracy performance in $\mathrm{E}+\mathrm{F}$ is working well under influence of up to $20 \%$ packet loss. It is getting the performance as bad as DR when dealing with $30 \%$ and above rates of packet loss. Although the performance of TSP is getting bad as the variable delay is getting higher, the overall performance of TSP is still shown to be the least position discrepancy in both maximum and average as compared to DR and E+F. Furthermore, Figure 7 has also clearly pointed out how accurate are the techniques used in position data prediction among the TS. For example in TS15 which the setting of network impairments are $20 \%$ of packet loss and variable delay from $50 \mathrm{~ms}$ to $100 \mathrm{~ms}$, the peak and average position discrepancy from the desired position using DR are $1.9^{\circ}$ and $0.2^{\circ}$ angular degree, while $\mathrm{E}+\mathrm{F}$ are $1.6^{\circ}$ and $0.14^{\circ}$ and TSP are $0.95^{\circ}$ and $0.14^{\circ}$. Figure 8 indicates the quantitative comparison of prediction techniques with the aspect of smoothness consistency in haptic position update. Figure 8 shows that the smoothness of haptic position update using DR always has the biggest gap between the position updates. The performance of $\mathrm{E}+\mathrm{F}$ in average of update gap is working well under influence of up to $20 \%$ packet loss. It is getting the performance as bad as DR when dealing with $30 \%$ and above rates of packet loss. The performance of TSP is shown the best performance in both maximum and average of position update gap. With the concern of maximum of update gap throughout the TS, E+F and TSP are capable to control below $1.7^{\circ}$ and $1.1^{\circ}$ angular degree respectively while DR is up to $3.1^{\circ}$. The larger value of the gap in position update, the less smooth of haptic positional interaction. On the other hand, the smoothness update of position is very subjective to individual of human perception, but assumed that the JND on position update is around $1^{\circ}$ angular degree gap which will trigger the human perception in noticing the unsmoothness of position update. The maximum of threshold of network impairments for TSP is able to undertake variable delay from $100 \mathrm{~ms}$ to $200 \mathrm{~ms}$, while $\mathrm{E}+\mathrm{F}$ can undertake from $50 \mathrm{~ms}$ to $100 \mathrm{~ms}$ and DR only from $20 \mathrm{~ms}$ to $50 \mathrm{~ms}$.

Moreover, Figure 9 and Figure 10 are to present the quantitative analysis in terms of accuracy and smoothness in haptic force update based on the three techniques of haptic prediction. Figure 9 shows the comparison of prediction techniques with the aspect of accuracy in predicted force data. With both maximum and average discrepancy in predicted force data, TSP has always shown as the best performance among all the TS, following by $\mathrm{E}+\mathrm{F}$ and the DR has the worst. With the unstable network connection as simulated based on TS24 which the setting of network impairments consists of variable delay range from $100 \mathrm{~ms}$ to $200 \mathrm{~ms}$ and $50 \%$ of packet loss, TSP is able to maintain the maximum and average force discrepancy from desired force in $0.48 \mathrm{~N}$ and $0.076 \mathrm{~N}$ of force respectively while $\mathrm{DR}$ is 0.68 and $\mathrm{N} 0.082 \mathrm{~N}$; $\mathrm{E}+\mathrm{F}$ is $0.64 \mathrm{~N}$ and $0.079 \mathrm{~N}$. Figure 10 presents the quantitative comparison of prediction techniques with the aspect of smoothness consistency in force update. With both maximum and average gap of force data update, TSP has always shown as the best performance in all the TS, following by E+F and the DR has the worst performance. If the JND on force update is assumed as $0.06 \mathrm{~N}$ that will trigger the human perception in noticing perceptible vibration, TSP is able to undertake the network impairment up to variable delay range from $100 \mathrm{~ms}$ to $200 \mathrm{~ms}$ with $40 \%$ of packet loss, while $\mathrm{E}+\mathrm{F}$ can undertake up to variable delay range from $20 \mathrm{~ms}$ to $50 \mathrm{~ms}$ and $20 \%$ of packet loss. However, DR cannot maintain the JND value unless no network impairment. 
TABLE 1. DifFERENT ATTRIBUTES OF VARIABLE DELAY WITH JITTER SETTING AND RATE OF PACKET LOSS IN TESTING SCENARIOS

\begin{tabular}{|c|c|c|c|c|c|c|c|c|c|c|}
\hline \multicolumn{3}{|c|}{ Jitter Settings } & \multicolumn{2}{|c|}{ Variable Delay } & \multicolumn{6}{|c|}{ Packet Loss (\% of 1000 packet per second Transmission rate ) } \\
\hline Alpha & Beta & Constant & $\operatorname{Min}(\mathrm{ms})$ & Max (ms) & 0 & 10 & 20 & 30 & 40 & 50 \\
\hline 1 & 19 & 0 & 1 & 20 & TS1 & TS2 & TS3 & TS4 & TS5 & TS6 \\
\hline 0 & 30 & 20 & 20 & 50 & TS7 & TS8 & TS9 & TS10 & TS11 & TS12 \\
\hline 0 & 50 & 50 & 50 & 100 & TS13 & TS14 & TS15 & TS16 & TS17 & TS18 \\
\hline 0 & 100 & 100 & 100 & 200 & TS19 & TS20 & TS21 & TS22 & TS23 & TS24 \\
\hline
\end{tabular}

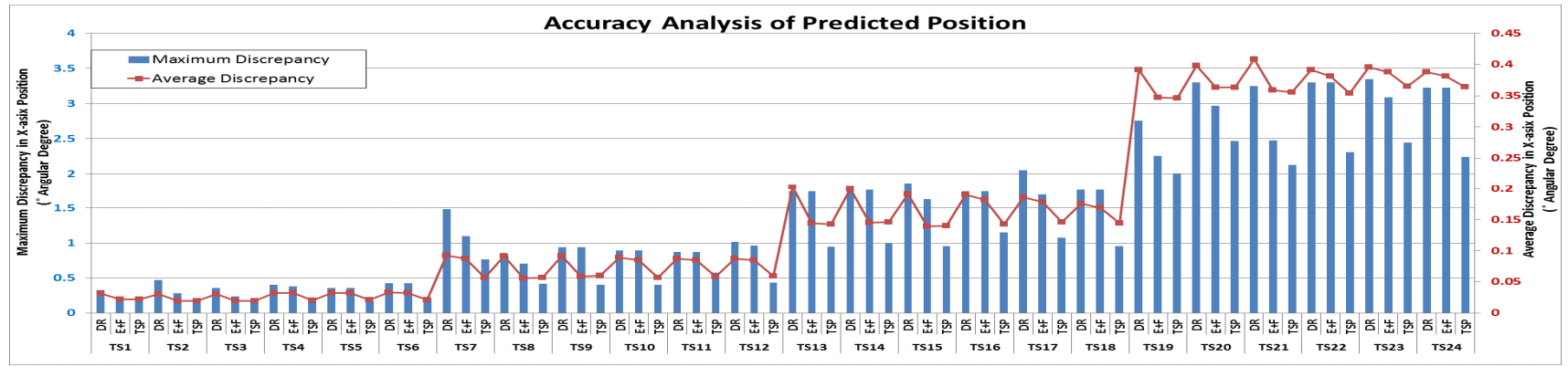

Figure 7. Comparison of prediction techniques with the aspect of accuracy in position data.

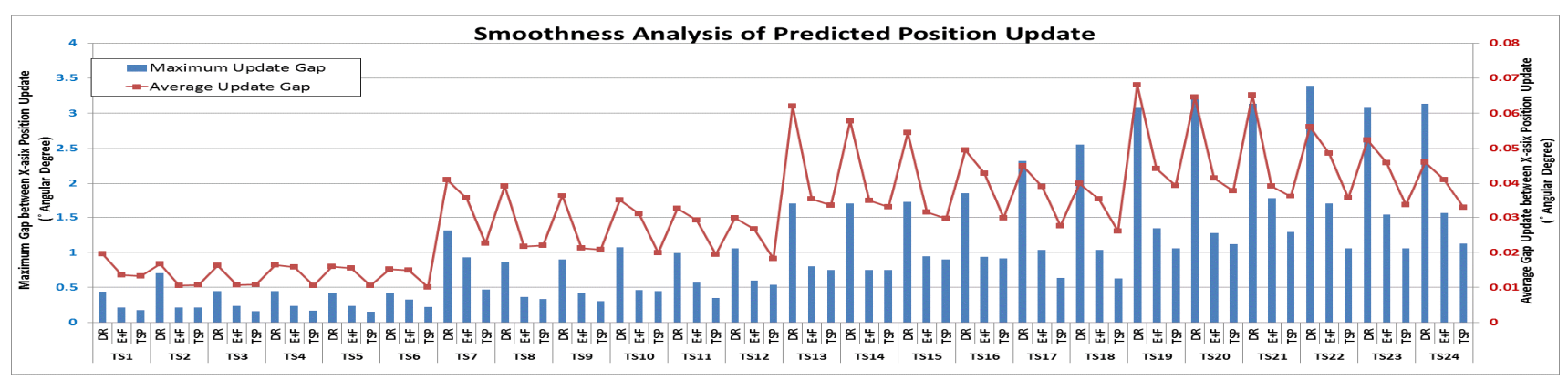

Figure 8. Comparison of prediction techniques with the aspect of smoothness consistency in position update.

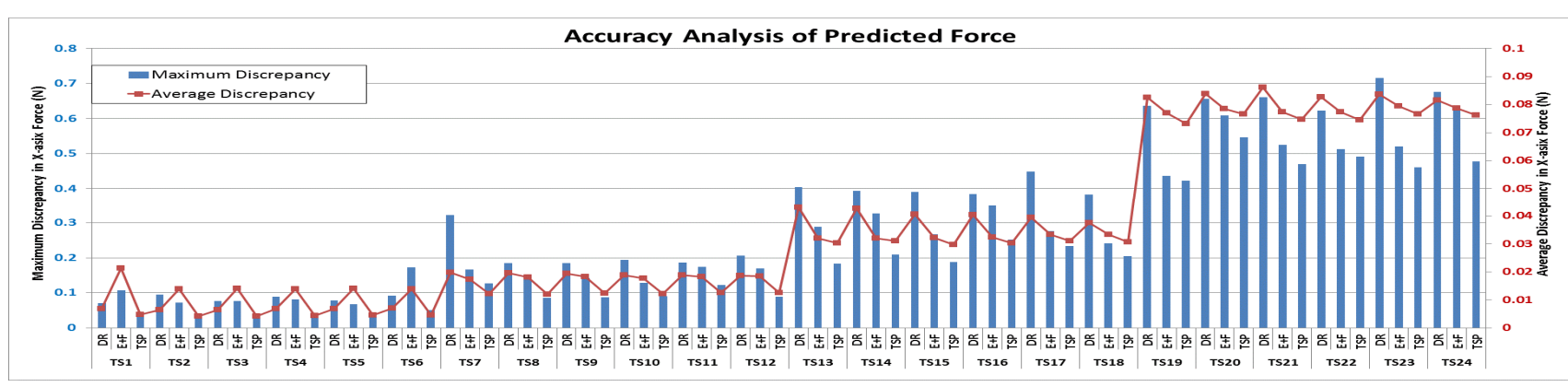

Figure 9. Comparison of prediction techniques with the aspect of accuracy in force data.

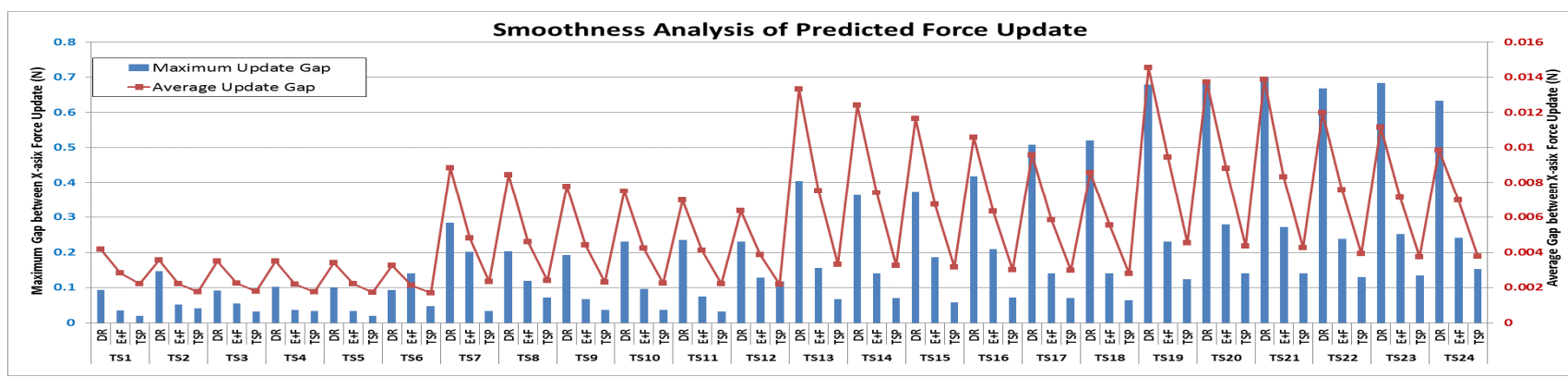

Figure 10. Comparison of prediction techniques with the aspect of smoothness consistency in force update. 


\section{VI.CONCLUSIONS}

A new predictive approach applied in haptic data, named as Trust Strategy Prediction (TSP) is introduced in this paper. As well as an evaluation applying the quantitative model is presented to analysis the performance in term of accuracy and smoothness consistency of haptic update as compared two well-known haptic data predictor: Dead Reckoning (DR) and velocity/yank estimation with filtering mechanism $(\mathrm{E}+\mathrm{F})$. Moreover, it is worth to noting the advantage of using quantitative analysis in haptic data prediction is able to easily identify the maximum threshold of network impairments for just-noticeable different (JND) in stability of haptic interaction. The results as shown in the quantitative analysis have proven performance of TSP is better through the comparison with DR and E+F and in terms of accuracy and smoothness updates. Furthermore, TSP is capable to undertake with the larger threshold of network impairments as compared to DR and $\mathrm{E}+\mathrm{F}$ while maintaining the smoothness of haptic update.

\section{ACKNOWLEDGMENT}

This work was financially supported by the Exploratory Research Grant Scheme (Grant No. ERGS/1/2012/TK06/ SYUC/02/3) by Ministry of Higher Education in Malaysia

\section{REFERENCES}

[1] M. Vu and U. Na, "A new 6-DOF haptic device for teleoperation of 6DOF serial robots," IEEE Transactions on Instrumentation and Measurement, vol. 60, no. 11, pp. 3510-3523, Nov. 2011.

[2] P. Hinterseer and E. Steinbach, "A Psychophysically Motivated Compression Approach for 3D Haptic Data," in IEEE Haptics Symposium, 2006, pp. 35-41.

[3] A. Marshall, K. M. Yap, and W. Yu, "Providing QoS for Networked Peers in Distributed Haptic Virtual Environments," Advances in Multimedia, vol. 2008, pp. 1-14, 2008.

[4] G. Kokkonis and K. Psannis, "Evaluating transport and application layer protocols for haptic applications," in IEEE International Workshop on Haptic Audio Visual Environments and Games, 2012, pp. 66-71.

[5] S. Islam, X. Liu, and A. El Saddik, "Teleoperation Systems With Symmetric and Unsymmetric Time Varying Communication Delay," IEEE Transactions on Instrumentation and Measurement, vol. 62, no. 11, pp. 2943-2953, 2013.

[6] J. Qin, K. Choi, R. Xu, P. WM, and H. PA, "Packet-loss-resilient perception-based haptic data reduction and transmission using ACK packets," in IEEE 11th International Conference on Signal Processing, 2012, pp. 1-6.

[7] M. Eid, J. Cha, and A. El Saddik, "Admux: An adaptive multiplexer for haptic-audio-visual data communication," IEEE Transactions on Instrumentation and Measurement, vol. 60, no. 1, pp. 21-31, 2011.
[8] H. J. Choi and S. Jung, "Compensation for time delay in tele-operation control of mobile robot using neural network," in IEEE 8th World Congress on Intelligent Control and Automation, 2010, pp. 1343-1347.

[9] V. Nitsch, B. Farber, L. Geiger, P. Hinterseer, and E. Steinbach, "An experimental study of lossy compression in a real telepresence and teleaction system," in Haptic Audio Visual Environments and their Applications, 2008, no. October, pp. 18-19.

[10] Y. You and M. Sung, "Haptic data transmission based on the prediction and compression," in IEEE International Conference on Communications, 2008, pp. 1824-1828.

[11] Y. Kusunose, Y. Ishibashi, N. Fukushima, and S. Sugawara, "Adaptive delta-causality control with prediction in networked real-time game using haptic media," in IEEE18th Asia-Pacific Conference on Communications (APCC), 2012, pp. 800-805.

[12] J. Lee and S. Payandeh, "Stability of internet-based teleoperation systems using Bayesian predictions," in IEEE World Haptics Conference, 2011, pp. 499-504.

[13] N. Sakr and J. Zhou, "Prediction-based haptic data reduction and transmission in telementoring systems," IEEE Transactions on Instrumentation and Measurement, vol. 58, no. 5, pp. 1727-1736, 2009.

[14] N. Sakr, J. Zhou, N. D. Georganas, J. Zhao, and X. Shen, "Predictionbased Haptic Data Reduction and Compression in Tele-Mentoring Systems," in 2008 IEEE Instrumentation and Measurement Technology Conference, 2008, pp. 1828-1832.

[15] T. H. Tee, K. S. Eu, K. M. Yap, A. Marshall, and T. Lee, "A Networkadaptive Compensation Technique for Tele-haptics using Position Prediction Algorithm," in IEEE International Symposium on Haptic Audio-Visual Environments and Games, 2013.

[16] K. M. Yap, A. Marshall, and W. Yu, "Supporting haptic collaboration across networked peers with real time force feedback in distributed virtual environments," in IEEE International Conference on Telecommunications and Malaysia International Conference on Communications, 2007, no. May, pp. 494-499.

[17] S. Lee, S. Moon, and J. Kim, "A network-adaptive transport scheme for haptic-based collaborative virtual environments," in 5th Workshop on Network and System Support for Games, 2006, pp. 1-8.

[18] S. Lee and J. Kim, "Dynamic network adaptation scheme employing haptic event priority for collaborative virtual environments," in ImmersCom, 2007.

[19] H. Al Osman, M. Eid, and A. El Saddik, "Evaluating ALPHAN: A communication protocol for haptic interaction," in IEEE Symposium on Haptic Interfaces for Virtual Environments and Teleoperator Systems, 2008, pp. 361-366.

[20] H. Al Osman, M. Eid, and A. El Saddik, "Evaluating ALPHAN with Multi-user Collaboration," in 12th IEEE/ACM International Symposium on Distributed Simulation and Real-Time Applications, 2008, pp. 181-186.

[21] Reza N. Jazar, Advanced Dynamics: Rigid Body, Multibody, and Aerospace Applications. John Wiley \& Sons, Inc, 2011, p. 131.

[22] Geomagic, "Geomagic Touch," Geomagic, 2013. [Online]. Available: www.geomagic.com/en/products/phantom-omni/overview. [Accessed: 10-Dec-2013].

[23] ZTI, "NetDisturb - Impairment Emulator Software for IP Networks (IPv4 \& IPv6)," ZTI Telecom, 2013. [Online]. Available: www.ztitelecom.com/EN/NetDisturb.html. [Accessed: 10-Dec-2013]. 